\title{
Diverse Soliton Structures for Fractional Nonlinear Schrodinger Equation, KdV Equation and WBBM Equation Adopting a New Technique
}

\section{Md. Tarikul Islam}

Hajee Mohammad Danesh Science and Technology University

Francisco Gomez ( $\sim$ jgomez@cenidet.edu.mx )

Centro Nacional de Investigacion y Desarrollo Tecnologico https://orcid.org/0000-0001-9403-3767

\section{Md. Ali Akbar}

University of Rajshahi

\section{Research Article}

Keywords: Improved auxiliary equation technique, wave variable transformation, fractional order nonlinear evolution equation, exact solution, soliton

Posted Date: July 9th, 2021

DOl: https://doi.org/10.21203/rs.3.rs-655487/v1

License: (c) (i) This work is licensed under a Creative Commons Attribution 4.0 International License. Read Full License 


\title{
Diverse soliton structures for fractional nonlinear Schrodinger equation, KdV equation and WBBM equation adopting a new technique
}

\author{
Md. Tarikul Islam ${ }^{1}$, J.F. Gómez-Aguilar ${ }^{2 *}$ Md. Ali Akbar ${ }^{3}$ \\ ${ }^{1}$ Department of Mathematics, Hajee Mohammad Danesh Science and Technology University, \\ Dinajpur, Bangladesh. \\ ${ }^{4}$ CONACyT-Tecnológico Nacional de México/CENIDET, Interior Internado Palmira S/N, \\ Col. Palmira, C.P. 62490, Cuernavaca, Morelos, México. \\ ${ }^{3}$ Department of Applied Mathematics, University of Rajshahi, Rajshahi, Bangladesh.
}

*Corresponding Author: jose.ga@ cenidet.tecnm.mx

\begin{abstract}
Nonlinear fractional order partial differential equations standing for the numerous dynamical systems relating to nature world are supposed to by unraveled for depicting complex physical phenomena. In this exploration, we concentrate to disentangle the space and time fractional nonlinear Schrodinger equation, Korteweg-De Vries (KdV) equation and the
\end{abstract} Wazwaz-Benjamin-Bona-Mahony (WBBM) equation bearing the noteworthy significance in accordance to their respective position. A composite wave variable transformation with the assistance of conformable fractional derivative transmutes the declared equations to ordinary differential equations. A successful implementation of the proposed improved auxiliary equation technique collects enormous wave solutions in the form of exponential, rational, trigonometric and hyperbolic functions. The found solutions involving many free parameters under consideration of particular values are figured out which appeared in different shape as kink type, anti-kink type, singular kink type, bell shape, anti-bell shape, singular bell shape, cuspon, peakon, periodic etc. The performance of the proposed scheme shows its potentiality through construction of fresh and further general exact traveling wave solutions of three nonlinear equations. A comparison of the achieved outcomes in this investigation with the results found in the literature ensures the diversity and novelty of ours. Consequently, the improved auxiliary equation technique stands as efficient and concise tool which deserves 
further use to unravel any other nonlinear evolution equations arise in various physical sciences like applied mathematics, mathematical physics and engineering.

Keywords: Improved auxiliary equation technique; wave variable transformation; fractional order nonlinear evolution equation; exact solution; soliton.

\section{Mathematics Subject Classifications: 35C08, 35R11}

\section{Introduction}

The nature is full of nonlinear wonders which has become an important matter of fact to be disclosed by the scholars and researchers day by day. These phenomena are modeled through the nonlinear partial differential equations which play significant roles to depict the underlying mechanisms of numerous complex physical phenomena relating to real world problems. These equations formulate noteworthy phenomena arise in applied sciences such as applied mathematics, mathematical physics, solid state physics, fluid mechanics, biology, solid state biology, chemistry, electric control theory, system identification, chaotic dynamical behavior of dynamical systems, signal processing, quasi-chaotic dynamical systems, food engineering, hydrodynamics, economics, finance and diffusion models [1-6]. Consequently, mathematicians and physicists pay deep attention to unravel the above models through the formulation of their solutions in approximate and appropriate form by computational and analytical techniques. A few methods to investigate nonlinear partial differential equations are available in ref. [7-21]. But no technique is unique to examine exact traveling wave solutions to nonlinear evolution equations. That is why, experts are hunting new methods incessantly. Khater et al. (2017) have introduced Khater method and found abundant elliptic and solitary wave solutions of three nonlinear equations [22]. This method has been applied by Bibi et al. (2017) to nonlinear Sharma Tasso-Olever (STO) equation of fractional order and found exact analytic solutions [23]. Later, new auxiliary equation method, modified auxiliary equation method and 
generalized Khater method mostly relevant to Khater method have been presented for analytic wave solutions to different nonlinear partial differential equations [24-31].

Subsequently, we are inspired and announced improved auxiliary equation technique as more general case of Khater method. The proposed method is employed to unravel the fractional nonlinear Schrodinger equation, KdV equation and WBBM equation and abundant soliton and other types solutions are successfully gained. The literature carries out a lot of work with the equations we aim to explore. Instantly, the fractional nonlinear Schrodinger equation has been demonstrated by Hemida et al., (2012) via homotopy analysis method and found few series solutions [32]; the same equation for $\alpha=1$ has been considered by Kaplan et al. (2016) and found three analytic solutions only by using the $\left(G^{\prime} / G, 1 / G\right)$-expansion method and one solution by the $\left(1 / G^{\prime}\right)$-expansion method [33]; arbitrary order nonlinear Schrodinger equation has been studied through fractional Riccati expansion method and achieved four exact wave solutions by Salam et al. [34]; the new type F-expansion method has been applied to construct exact solutions of the space-time fractional cubic Schrodinger equation by Pandir and Duzgun [35]; Wazwaz and Kaur have used variational iteration method and improved $\left(G^{\prime} / G\right)$ expansion method which left several solutions to the integer order cubic Schrodinger equation [36]; Cheema and Younis have considered the same equation for $\alpha=1$ and obtained sixteen solutions by the extended Fan sub-equation method [37]; Akbar et al. (2019) have investigated the equation of integer order by new auxiliary equation method and found soliton types solutions [38]. The conformable space-time fractional KdV equation has been studied by Yaslan and Girgin (2019) via the $\left(G^{\prime} / G^{2}\right)$-expansion method for analytic wave solutions [39]; Odibat (2017) has employed Riccati equation approach to the KdV equation and constructed wave solutions [40]; the local variational iteration method and the local fractional series expansion method has been adopted by Jasim and Baleanu (2019) to study the fractional KdV equation [41]; Liu and Zhang (2018) have investigated the same equation by improved $\left(G^{\prime} / G\right)$ - 
expansion method and found exact analytic solutions [42]; Jacobi elliptic function expansion method has been assumed to examine the KdV equation by Dascioglu et al. (2017) [43]. Akram et al. (2021) have studied the fractional Wazwaz-Benjamin-Bona-Mahony by extended modified auxiliary equation mapping method which left different soliton types solutions [44]; the same equation has been explored by Seadawy et al. (2019) with the help of simple ansatzs and gained hyperbolic function and periodic function solutions [45].

The suggested equations involving conformable fractional derivatives are converted into the differential equations of single variable by means of a composite transformation of wave variable. Khalil et al. have proclaimed the conformable fractional derivative as follows [46]:

The conformable derivative of a function $\Phi(x)$ is

$$
D_{x}^{\alpha}(\Phi(x))=\lim _{\varepsilon \rightarrow 0} \frac{\Phi\left(x+\varepsilon x^{1-\alpha}\right)-\Phi(x)}{\varepsilon},
$$

where $x>0$ and $\alpha$ denotes the order of derivative such as $0<\alpha \leq 1$. The properties of this definition are brought out by the following theorems:

Theorem 1. For $x>0, \alpha \in(0,1]$, the functions $\Phi(x)$ and $\Psi(x)$ are considered to be $\alpha$ differentiable. Then

(a) $D_{x}^{\alpha}\left(x^{n}\right)=n x^{n-\alpha} \forall n \in \boldsymbol{R}$.

(b) $D_{x}^{\alpha}(\lambda)=0$, where $\lambda$ is any constant.

(c) $D_{x}^{\alpha}(a \Phi(x)+b \Psi(x))=a D_{x}^{\alpha}(\Phi(x))+b D_{x}^{\alpha}(\Psi(x)) \forall a, b \in \boldsymbol{R}$.

(d) $D_{x}^{\alpha}(\Phi(x) \Psi(x))=\Phi(x) D_{x}^{\alpha}(\Psi(x))+\Psi(x) D_{x}^{\alpha}(\Phi(x))$.

(e) $D_{x}^{\alpha}(\Phi(x) / \Psi(x))=\frac{\Psi(x) D_{x}^{\alpha}(\Phi(x))-\Phi(x) D_{x}^{\alpha}(\Psi(x))}{\Psi^{2}(x)}$.

(f) if $\Phi$ is differentiable, then $D_{x}^{\alpha}(\Phi)(x)=x^{1-\alpha} \frac{d \Phi(x)}{d x}$.

Theorem 2: Let an $\alpha$-differentiable and also differentiable function be $\Phi(x)$ for $0<\alpha \leq 1$ in which another function $\Psi(x)$ is also supposed to be differentiable, then

$$
D_{x}^{\alpha}(\Phi(x) . \Psi(x))=x^{1-\alpha} \Psi^{\prime}(x) \Phi^{\prime}(\Psi(x)) .
$$


This paper makes available abundant distinct and novel exact traveling wave solutions. Finally, we bring out a comparable study of the gained outcomes with the existing results in the literature which ensures the newness and generality of our well-furnished solutions. This work might encourage the scholars for further study and create a landmark in the research area.

\section{Elucidation of the proposed improved auxiliary equation technique}

We take into account the following type non-integer order nonlinear evolution equation:

$$
P\left(u, D_{t}^{\alpha} u, D_{x}^{\alpha} u, D_{t t}^{2 \alpha} u, D_{x x}^{2 \alpha} u, D_{t}^{\alpha} D_{x}^{\alpha} u, \ldots \ldots \ldots\right)=0 \text {, where } 0<\alpha \leq 1,
$$

which stands with a polynomial $P$ involving the function $u$ and it's several fractional order partial derivatives.

Introduce the compound wave variable alteration

$$
u=u(x, t)=U(\xi), \xi=\xi(x, t)
$$

Making usage of Eq. (2.2) coverts Eq. (2.1) to the following ODE due to the variable $\xi$ :

$$
F\left(U, U^{\prime}, U^{\prime \prime}, U^{\prime \prime \prime}, \ldots \ldots \ldots\right)=0
$$

Integrate Eq. (2.3) as much possible and the constant of integration can be diminished as we desire to construct soliton types solutions.

The procedures of the proposed technique are as follows:

The solution of Eq. (2.1) is supposed to be in the form

$$
u(\xi)=\frac{\sum_{i=0}^{n} c_{i} a^{i \phi(\xi)}}{\sum_{i=0}^{n} d_{i} a^{i \phi(\xi)}}
$$

where $c_{i}{ }^{\prime} s$ and $d_{i}{ }^{\prime} s$ are unknown parameters; $n$ is determined by applying homogeneous balance to Eq. (2.3) and the function $\phi(\xi)$ stands for the solution of the following auxiliary equation: 


$$
\phi^{\prime}(\xi)=\frac{1}{\ln a}\left\{p a^{-\phi(\xi)}+q+r a^{\phi(\xi)}\right\}
$$

Absorbing the value of $n$ determined above, Eq. (2.4) with its necessary derivatives and Eq. (2.5) forces the left-hand side of Eq. (2.3) to be a polynomial. Solving each coefficient after assigning zero with the aid of computational software Maple yields the values of necessary parameters. Inserting these values and the solutions of Eq. (2.5) in Eq. (2.4) provides the anticipated solutions of Eq. (2.1). The solutions of Eq. (2.5) are available in [38].

Now, we formulate the closed form wave solutions of the following space and time fractional nonlinear partial differential equations:

\section{The nonlinear Schrodinger equation of fractional order}

Consider the space and time fractional nonlinear Schrodinger equation

$$
i D_{t}^{\alpha} u+\delta D_{x}^{2 \beta} u+b|u|^{2} u=0, t>0,0<\alpha, \beta \leq 1
$$

where $b$ and $\delta$ are non-zero real constants; $x$ is the spatial variable and $t$ represents time [35]. This equation occurs in plasma physics, non-linear optics and superconductivity. We introduce the fractional composite transformation as follows:

$$
u(x, t)=e^{i \psi} v(\xi), \psi=\frac{l x^{\beta}}{\beta}+\frac{m t^{\alpha}}{\alpha}, \xi=k\left(\frac{x^{\beta}}{\beta}-\frac{2 l t^{\alpha}}{\alpha}\right),
$$

where $l, k$ and $m$ are free parameters to be determined later. Eq. (3.1) with the aid of Eq. (3.2) reduces to the ODE

$$
i(-2 k l+2 a k l) v^{\prime}-\left(a l^{2}+m\right) v+a k^{2} v^{\prime \prime}+b v^{3}=0 .
$$

The imaginary part yields $a=1$ and hence the real part becomes

$$
-\left(l^{2}+m\right) v+k^{2} v^{\prime \prime}+b v^{3}=0 .
$$

Homogenous balance technique due to $v^{\prime \prime}$ and $v^{3}$ forces Eq. (...) to be 


$$
u(\xi)=\frac{c_{0}+c_{1} a^{\phi(\xi)}}{d_{0}+d_{1} a^{\phi(\xi)}}
$$

Eq. (3.5) alongside its necessary derivatives and Eq. (2.5) reduces Eq. (3.4) to a polynomial in $a^{\phi(\xi)}$. Assigning each coefficient to zero and solving by computational software Maple provides the following results:

Case 1: $c_{0}=\mp \frac{\left(2 d_{1} p-q d_{0}\right) k}{\sqrt{-2 b}}, c_{1}=\mp \frac{\left(q d_{1}-2 r d_{0}\right) k}{\sqrt{-2 b}}, l= \pm \frac{1}{2} \sqrt{8 p r k^{2}-2 k^{2} q^{2}-4 m}$.

Case 2: $c_{0}=\mp \frac{2 d_{1} p k}{\sqrt{-2 b}}, c_{1}= \pm \frac{k q d_{1}}{\sqrt{-2 b}}, d_{0}=0, l= \pm \frac{1}{2} \sqrt{8 p r k^{2}-2 k^{2} q^{2}-4 m}$

Case 3: $c_{0}= \pm \frac{d_{0} k \sqrt{4 p r-q^{2}}}{\sqrt{-2 b}}, c_{1}= \pm \frac{d_{0} k\left(q^{2}-4 p r \pm q \sqrt{4 p r-q^{2}}\right)}{2 p \sqrt{-2 b}}, d_{1}=\frac{d_{0}\left(q \pm \sqrt{4 p r-q^{2}}\right)}{2 p}$

$$
l= \pm \frac{1}{2} \sqrt{8 p r k^{2}-2 k^{2} q^{2}-4 m}
$$

Case 4: $c_{0}=0, c_{1}= \pm \frac{k d_{1}\left(q^{2}-4 p r\right)}{q \sqrt{-2 b}}, d_{0}=\frac{2 p d_{1}}{q}, l= \pm \frac{1}{2} \sqrt{8 p r k^{2}-2 k^{2} q^{2}-4 m}$.

The above cases produce much more solutions to the fractional nonlinear Schrodinger equation which all seem to be absurd to record here. We think reasonable to deal with only one case and thereupon considering case 1 and the solutions of Eq. (2.5) the wave solutions are given as follows:

When $q^{2}-4 p r<0$ and $r \neq 0$,

$$
\begin{aligned}
& u_{1,2}(\xi)= \pm \frac{k e^{i \psi}}{\sqrt{-2 b}} \times \frac{2 r\left(2 d_{1} p-q d_{0}\right)-\left(q d_{1}-2 r d_{0}\right)\left(q-\sqrt{4 p r-q^{2}} \tan \left(\sqrt{4 p r-q^{2}} \xi / 2\right)\right)}{2 r d_{0}-d_{1}\left(q-\sqrt{4 p r-q^{2}} \tan \left(\sqrt{4 p r-q^{2}} \xi / 2\right)\right)} \\
& u_{3,4}(\xi)= \pm \frac{k e^{i \psi}}{\sqrt{-2 b}} \times \frac{2 r\left(2 d_{1} p-q d_{0}\right)-\left(q d_{1}-2 r d_{0}\right)\left(q+\sqrt{4 p r-q^{2}} \cot \left(\sqrt{4 p r-q^{2}} \xi / 2\right)\right)}{2 r d_{0}-d_{1}\left(q+\sqrt{4 p r-q^{2}} \cot \left(\sqrt{4 p r-q^{2}} \xi / 2\right)\right)}
\end{aligned}
$$

where $\xi=k\left(\frac{x^{\beta}}{\beta}-\frac{2 l t^{\alpha}}{\alpha}\right), \psi=\frac{l x^{\beta}}{\beta}+\frac{m t^{\alpha}}{\alpha}$ with $l= \pm \frac{1}{2} \sqrt{2 k^{2}\left(4 p r-q^{2}\right)-4 m}$

According to the condition $q^{2}-4 p r>0$ and $r \neq 0$, 


$$
\begin{aligned}
& u_{5,6}(\xi)= \pm \frac{k e^{i \psi}}{\sqrt{-2 b}} \times \frac{2 r\left(2 d_{1} p-q d_{0}\right)-\left(q d_{1}-2 r d_{0}\right)\left(q+\sqrt{q^{2}-4 p r} \tanh \left(\sqrt{q^{2}-4 p r} \xi / 2\right)\right)}{2 r d_{0}-d_{1}\left(q+\sqrt{q^{2}-4 p r} \tanh \left(\sqrt{q^{2}-4 p r} \xi / 2\right)\right)}, \\
& u_{7,8}(\xi)= \pm \frac{k e^{i \psi}}{\sqrt{-2 b}} \times \frac{2 r\left(2 d_{1} p-q d_{0}\right)-\left(q d_{1}-2 r d_{0}\right)\left(q+\sqrt{q^{2}-4 p r} \operatorname{coth}\left(\sqrt{q^{2}-4 p r} \xi / 2\right)\right)}{2 r d_{0}-d_{1}\left(q+\sqrt{q^{2}-4 p r} \operatorname{coth}\left(\sqrt{q^{2}-4 p r} \xi / 2\right)\right)},
\end{aligned}
$$

where $\xi=k\left(\frac{x^{\beta}}{\beta}-\frac{2 l t^{\alpha}}{\alpha}\right), \psi=\frac{l x^{\beta}}{\beta}+\frac{m t^{\alpha}}{\alpha}$ with $l= \pm \frac{1}{2} \sqrt{8 p r k^{2}-2 k^{2} q^{2}-4 m}$.

For $q^{2}+4 p^{2}<0, r \neq 0$ and $r=-p$,

$$
\begin{gathered}
u_{9,10}(\xi)= \pm \frac{k e^{i \psi}}{\sqrt{-2 b}} \times \frac{2 p\left(2 d_{1} p-q d_{0}\right)+\left(q d_{1}+2 p d_{0}\right)\left(q-\sqrt{-q^{2}-4 p^{2}} \tan \left(\sqrt{-q^{2}-4 p^{2}} \xi / 2\right)\right)}{2 p d_{0}+d_{1}\left(q-\sqrt{-q^{2}-4 p^{2}} \tan \left(\sqrt{-q^{2}-4 p^{2}} \xi / 2\right)\right)} \\
u_{11,12}(\xi)= \pm \frac{k e^{i \psi}}{\sqrt{-2 b}} \times \frac{2 p\left(2 d_{1} p-q d_{0}\right)+\left(q d_{1}+2 p d_{0}\right)\left(q+\sqrt{-q^{2}-4 p^{2}} \cot \left(\sqrt{-q^{2}-4 p^{2}} \xi / 2\right)\right)}{2 p d_{0}+d_{1}\left(q+\sqrt{-q^{2}-4 p^{2}} \cot \left(\sqrt{-q^{2}-4 p^{2}} \xi / 2\right)\right)}
\end{gathered}
$$

where $\xi=k\left(\frac{x^{\beta}}{\beta}-\frac{2 l t^{\alpha}}{\alpha}\right), \psi=\frac{l x^{\beta}}{\beta}+\frac{m t^{\alpha}}{\alpha}$ with $l= \pm \frac{1}{2} \sqrt{-8 p^{2} k^{2}-2 k^{2} q^{2}-4 m}$.

If $q^{2}+4 p^{2}>0, r \neq 0$ and $r=-p$,

$$
\begin{aligned}
& u_{13,14}(\xi)= \pm \frac{k e^{i \psi}}{\sqrt{-2 b}} \times \frac{2 p\left(2 d_{1} p-q d_{0}\right)+\left(q d_{1}+2 p d_{0}\right)\left(q+\sqrt{q^{2}+4 p^{2}} \tanh \left(\sqrt{q^{2}+4 p^{2}} \xi / 2\right)\right)}{2 p d_{0}+d_{1}\left(q+\sqrt{q^{2}+4 p^{2}} \tanh \left(\sqrt{q^{2}+4 p^{2}} \xi / 2\right)\right)} \\
& u_{15,16}(\xi)= \pm \frac{k e^{i \psi}}{\sqrt{-2 b}} \times \frac{2 p\left(2 d_{1} p-q d_{0}\right)+\left(q d_{1}+2 p d_{0}\right)\left(q+\sqrt{q^{2}+4 p^{2}} \operatorname{coth}\left(\sqrt{q^{2}+4 p^{2}} \xi / 2\right)\right)}{2 p d_{0}+d_{1}\left(q+\sqrt{q^{2}+4 p^{2}} \operatorname{coth}\left(\sqrt{q^{2}+4 p^{2}} \xi / 2\right)\right)}
\end{aligned}
$$

where $\xi=k\left(\frac{x^{\beta}}{\beta}-\frac{2 l t^{\alpha}}{\alpha}\right), \psi=\frac{l x^{\beta}}{\beta}+\frac{m t^{\alpha}}{\alpha}$ with $l= \pm \frac{1}{2} \sqrt{-8 p^{2} k^{2}-2 k^{2} q^{2}-4 m}$.

Once $q^{2}-4 p^{2}<0$ and $r=p$,

$$
\begin{aligned}
& u_{17,18}(\xi)= \pm \frac{k e^{i \psi}}{\sqrt{-2 b}} \times \frac{2 p\left(2 d_{1} p-q d_{0}\right)-\left(q d_{1}-2 p d_{0}\right)\left(q-\sqrt{4 p^{2}-q^{2}} \tan \left(\sqrt{4 p^{2}-q^{2}} \xi / 2\right)\right)}{2 p d_{0}+d_{1}\left(-q+\sqrt{4 p^{2}-q^{2}} \tan \left(\sqrt{4 p^{2}-q^{2}} \xi / 2\right)\right)} \\
& u_{19,20}(\xi)= \pm \frac{k e^{i \psi}}{\sqrt{-2 b}} \times \frac{2 p\left(2 d_{1} p-q d_{0}\right)-\left(q d_{1}-2 p d_{0}\right)\left(q+\sqrt{4 p^{2}-q^{2}} \cot \left(\sqrt{4 p^{2}-q^{2}} \xi / 2\right)\right)}{2 p d_{0}-d_{1}\left(q+\sqrt{4 p^{2}-q^{2}} \cot \left(\sqrt{4 p^{2}-q^{2}} \xi / 2\right)\right)}
\end{aligned}
$$

where $\xi=k\left(\frac{x^{\beta}}{\beta}-\frac{2 l t^{\alpha}}{\alpha}\right), \psi=\frac{l x^{\beta}}{\beta}+\frac{m t^{\alpha}}{\alpha}$ with $l= \pm \frac{1}{2} \sqrt{8 p^{2} k^{2}-2 k^{2} q^{2}-4 m}$. 
After $q^{2}-4 p^{2}>0$ and $r=p$,

$$
\begin{aligned}
& u_{21,22}(\xi)= \pm \frac{k e^{i \psi}}{\sqrt{-2 b}} \times \frac{2 p\left(2 d_{1} p-q d_{0}\right)-\left(q d_{1}-2 p d_{0}\right)\left(q+\sqrt{q^{2}-4 p^{2}} \tanh \left(\sqrt{q^{2}-4 p^{2}} \xi / 2\right)\right)}{2 p d_{0}-d_{1}\left(q+\sqrt{q^{2}-4 p^{2}} \tanh \left(\sqrt{q^{2}-4 p^{2}} \xi / 2\right)\right)} \\
& u_{23,24}(\xi)= \pm \frac{k e^{i \psi}}{\sqrt{-2 b}} \times \frac{2 p\left(2 d_{1} p-q d_{0}\right)-\left(q d_{1}-2 p d_{0}\right)\left(q+\sqrt{q^{2}-4 p^{2}} \operatorname{coth}\left(\sqrt{q^{2}-4 p^{2}} \xi / 2\right)\right)}{2 p d_{0}-d_{1}\left(q+\sqrt{q^{2}-4 p^{2}} \operatorname{coth}\left(\sqrt{q^{2}-4 p^{2}} \xi / 2\right)\right)}
\end{aligned}
$$

where $\xi=k\left(\frac{x^{\beta}}{\beta}-\frac{2 l t^{\alpha}}{\alpha}\right), \psi=\frac{l x^{\beta}}{\beta}+\frac{m t^{\alpha}}{\alpha}$ with $l= \pm \frac{1}{2} \sqrt{8 p^{2} k^{2}-2 k^{2} q^{2}-4 m}$.

Due to $q^{2}=4 p r$,

$$
u_{25,26}(\xi)= \pm \frac{k e^{i \psi}}{\sqrt{-2 b}} \times \frac{2 r\left(2 d_{1} p-q d_{0}\right) \xi-\left(q d_{1}-2 r d_{0}\right)(2+q \xi)}{2 d_{0} r \xi-d_{1}(2+q \xi)}
$$

where $\xi=k\left(\frac{x^{\beta}}{\beta}-\frac{2 l t^{\alpha}}{\alpha}\right), \psi=\frac{l x^{\beta}}{\beta}+\frac{m t^{\alpha}}{\alpha}$ with $l= \pm \sqrt{-m}$.

Under the assumption $r p<0, q=0$ and $r \neq 0$,

$$
\begin{aligned}
& u_{27,28}(\xi)= \pm \frac{2 k e^{i \psi}}{\sqrt{-2 b}} \times \frac{d_{1} p+r d_{0} \sqrt{-p / r} \tanh (\sqrt{-r p} \xi)}{d_{0}-d_{1} \sqrt{-p / r} \tanh (\sqrt{-r p} \xi)}, \\
& u_{29,30}(\xi)= \pm \frac{2 k e^{i \psi}}{\sqrt{-2 b}} \times \frac{d_{1} p+r d_{0} \sqrt{-p / r} \operatorname{coth}(\sqrt{-r p} \xi)}{d_{0}-d_{1} \sqrt{-p / r} \operatorname{coth}(\sqrt{-r p} \xi)}
\end{aligned}
$$

where $\xi=k\left(\frac{x^{\beta}}{\beta}-\frac{2 l t^{\alpha}}{\alpha}\right), \psi=\frac{l x^{\beta}}{\beta}+\frac{m t^{\alpha}}{\alpha}$ with $l= \pm \sqrt{2 p r k^{2}-m}$.

For $q=0$ and $p=-r$,

$$
u_{31,32}(\xi)= \pm \frac{2 r k e^{i \psi}}{\sqrt{-2 b}} \times \frac{d_{0}+d_{1}+\left(d_{0}-d_{1}\right) e^{2 r \xi}}{d_{0}+d_{1}+\left(d_{1}-d_{0}\right) e^{2 r \xi}}
$$

where $\xi=k\left(\frac{x^{\beta}}{\beta}-\frac{2 l t^{\alpha}}{\alpha}\right), \psi=\frac{l x^{\beta}}{\beta}+\frac{m t^{\alpha}}{\alpha}$ with $l= \pm \sqrt{-2 r^{2} k^{2}-m}$.

For the condition $p=q=K$ and $r=0$,

$$
u_{33,34}(\xi)= \pm \frac{k K e^{i \psi}}{\sqrt{-2 b}} \times \frac{-d_{0}+d_{1}\left(1+e^{K \xi}\right)}{d_{0}+d_{1}\left(e^{K \xi}-1\right)}
$$


where $\xi=k\left(\frac{x^{\beta}}{\beta}-\frac{2 l t^{\alpha}}{\alpha}\right), \psi=\frac{l x^{\beta}}{\beta}+\frac{m t^{\alpha}}{\alpha}$ with $l= \pm \frac{1}{2} \sqrt{-2 k^{2} K^{2}-4 m}$.

When $q=r=K$ and $p=0$,

$$
u_{35,36}(\xi)= \pm \frac{k K e^{i \psi}}{\sqrt{-2 b}} \times \frac{d_{1}\left(1-e^{K \xi}\right)-d_{0}\left(1+e^{K \xi}\right)}{d_{0}\left(1-e^{K \xi}\right)+d_{1} e^{K \xi}}
$$

where $\xi=k\left(\frac{x^{\beta}}{\beta}-\frac{2 l t^{\alpha}}{\alpha}\right), \psi=\frac{l x^{\beta}}{\beta}+\frac{m t^{\alpha}}{\alpha}$ with $l= \pm \frac{1}{2} \sqrt{-2 k^{2} K^{2}-4 m}$.

If $q=p+r$,

$$
u_{37,38}(\xi)= \pm \frac{k e^{i \psi}}{\sqrt{-2 b}} \times \frac{\left(2 d_{1} p-(p+r) d_{0}\right)\left(1-r e^{(p-r) \xi}\right)-\left((p+r) d_{1}-2 r d_{0}\right)\left(1-p e^{(p-r) \xi}\right)}{d_{0}\left(1-r e^{(p-r) \xi}\right)-d_{1}\left(1-p e^{(p-r) \xi}\right)},
$$

where $\xi=k\left(\frac{x^{\beta}}{\beta}-\frac{2 l t^{\alpha}}{\alpha}\right), \psi=\frac{l x^{\beta}}{\beta}+\frac{m t^{\alpha}}{\alpha}$ with $l= \pm \frac{1}{2} \sqrt{4 p r k^{2}-2 k^{2}\left(p^{2}+r^{2}\right)-4 m}$.

When $q=-(p+r)$,

$$
u_{39,40}(\xi)= \pm \frac{k e^{i \psi}}{\sqrt{-2 b}} \times \frac{\left(2 d_{1} p+(p+r) d_{0}\right)\left(r-e^{(p-r) \xi}\right)-\left((p+r) d_{1}+2 r d_{0}\right)\left(p-e^{(p-r) \xi}\right)}{\left(d_{0} r+d_{1} p\right)-\left(d_{0}+d_{1}\right) e^{(p-r) \xi}}
$$

where $\xi=k\left(\frac{x^{\beta}}{\beta}-\frac{2 l t^{\alpha}}{\alpha}\right), \psi=\frac{l x^{\beta}}{\beta}+\frac{m t^{\alpha}}{\alpha}$ with $l= \pm \frac{1}{2} \sqrt{4 p r k^{2}-2 k^{2}\left(p^{2}+r^{2}\right)-4 m}$.

Considering $p=0$,

$$
u_{41,42}(\xi)= \pm \frac{k e^{i \psi}}{\sqrt{-2 b}} \times \frac{\left(q d_{1}-2 r d_{0}\right) q e^{q \xi}-q d_{0}\left(1-r e^{q \xi}\right)}{d_{0}-\left(d_{0} r-d_{1} q\right) e^{q \xi}}
$$

where $\xi=k\left(\frac{x^{\beta}}{\beta}-\frac{2 l t^{\alpha}}{\alpha}\right), \psi=\frac{l x^{\beta}}{\beta}+\frac{m t^{\alpha}}{\alpha}$ with $l= \pm \frac{1}{2} \sqrt{-2 k^{2} q^{2}-4 m}$.

Along with $r=q=p \neq 0$,

$$
u_{43,44}(\xi)= \pm \frac{k e^{i \psi}}{\sqrt{-2 b}} \times \frac{2\left(2 d_{1} p-p d_{0}\right)+\left(p d_{1}-2 p d_{0}\right)\{\sqrt{3} \tan (\sqrt{3} p \xi / 2)-1\}}{2 d_{0}+d_{1}\{\sqrt{3} \tan (\sqrt{3} p \xi / 2)-1\}}
$$

where $\xi=k\left(\frac{x^{\beta}}{\beta}-\frac{2 l t^{\alpha}}{\alpha}\right), \psi=\frac{l x^{\beta}}{\beta}+\frac{m t^{\alpha}}{\alpha}$ with $l= \pm \frac{1}{2} \sqrt{6 p^{2} k^{2}-4 m}$.

Once $r=q=0$, 


$$
u_{45,46}(\xi)= \pm \frac{k e^{i \psi}}{\sqrt{-2 b}} \times \frac{2 d_{1} p}{d_{0}+d_{1} p \xi}
$$

where $\xi=k\left(\frac{x^{\beta}}{\beta}-\frac{2 l t^{\alpha}}{\alpha}\right), \psi=\frac{l x^{\beta}}{\beta}+\frac{m t^{\alpha}}{\alpha}$ with $l= \pm \sqrt{-m}$.

If we take $p=q=0$,

$$
u_{47,48}(\xi)= \pm \frac{k e^{i \psi}}{\sqrt{-2 b}} \times \frac{2 r d_{0}}{d_{0} r \xi-d_{1}}
$$

where $\xi=k\left(\frac{x^{\beta}}{\beta}-\frac{2 l t^{\alpha}}{\alpha}\right), \psi=\frac{l x^{\beta}}{\beta}+\frac{m t^{\alpha}}{\alpha}$ with $l= \pm \sqrt{-m}$.

According to the hypothesis $r=p$ and $q=0$,

$$
u_{49,50}(\xi)= \pm \frac{2 k p e^{i \psi}}{\sqrt{-2 b}} \times \frac{d_{1}-d_{0} \tan (p \xi)}{d_{0}+d_{1} \tan (p \xi)}
$$

where $\xi=k\left(\frac{x^{\beta}}{\beta}-\frac{2 l t^{\alpha}}{\alpha}\right), \psi=\frac{l x^{\beta}}{\beta}+\frac{m t^{\alpha}}{\alpha}$ with $l= \pm \sqrt{2 p^{2} k^{2}-m}$.

Due to the supposition $r=0$,

$$
u_{51,52}(\xi)= \pm \frac{k e^{i \psi}}{\sqrt{-2 b}} \times \frac{\left(2 d_{1} p-q d_{0}\right)+q d_{1}\left(e^{q \xi}-m / n\right)}{d_{0}+d_{1}\left(e^{q \xi}-m / n\right)}
$$

where $\xi=k\left(\frac{x^{\beta}}{\beta}-\frac{2 l t^{\alpha}}{\alpha}\right), \psi=\frac{l x^{\beta}}{\beta}+\frac{m t^{\alpha}}{\alpha}$ with $l= \pm \frac{1}{2} \sqrt{-2 k^{2} q^{2}-4 m}$.

Making a comparable study of the found solutions to those existing ever it is claimed that this study ensures the diversity and novelty of our results [32-38].

\section{The space and time fractional nonlinear KdV equation}

The KdV equation of integer order was first derived by Boussinesq in 1877 and then Diederik Korteweg and Gustav de Vries had developed it in 1895 [39]. This equation stands for illustrating surface waves of small amplitude and long wavelength on shallow water and inner waves in a shallow density-stratified liquid. The fractional $\mathrm{KdV}$ model is taken the following form [42]: 


$$
D_{t}^{\alpha} u+\delta u D_{x}^{\beta} u+b D_{x}^{3 \beta} u=0,0<\alpha \leq 1,0<\beta \leq 1
$$

where $\delta$ and $b$ are non-zero real parameters. Eq. (4.1) with the aid of transformation

$$
u(x, t)=u(\xi), \xi=\frac{k t^{\alpha}}{\alpha}+\frac{m x^{\beta}}{\beta}
$$

turns into ODE as

$$
k u^{\prime}+\delta m u u^{\prime}+b m^{3} u^{\prime \prime \prime}=0
$$

Taking anti-derivative with zero integral constant gives

$$
k u+\frac{\delta m u^{2}}{2}+b m^{3} u^{\prime \prime}=0
$$

The solution of Eq. (4.4) under the balancing theme is of the form

$$
u(\xi)=\frac{c_{0}+c_{1} a^{\phi(\xi)}+c_{2} a^{2 \phi(\xi)}}{d_{0}+d_{1} a^{\phi(\xi)}+d_{2} a^{2 \phi(\xi)}}
$$

Eq. (4.5) together second derivative and Eq. (2.5) reduces the left side of Eq. (4.4) to a polynomial in $a^{\phi(\xi)}$. Collecting the coefficients and setting to zero yields a system of algebraic equations. Solve this system for the following outcomes:

Case 1: $c_{0}=-\frac{12 b d_{0} m^{2} p r}{\delta}, c_{1}=-\frac{12 b d_{0} m^{2} q r}{\delta}, c_{2}=-\frac{12 b d_{0} m^{2} r^{2}}{\delta}$,

$$
d_{1}=d_{2}=0, k=b m^{3}\left(4 p r-q^{2}\right)
$$

Case 2: $c_{0}=-\frac{12 b d_{2} m^{2} p^{2}}{\delta}, c_{1}=-\frac{12 b d_{2} m^{2} p q}{\delta}, c_{2}=-\frac{12 b d_{2} m^{2} p r}{\delta}$,

$$
d_{0}=d_{1}=0, k=b m^{3}\left(4 p r-q^{2}\right)
$$

Case 3: $c_{0}=-\frac{12 b d_{2} m^{2} p^{2}}{\delta}, c_{1}=-\frac{12 b d_{2} m^{2} p q}{\delta}, c_{2}=-\frac{2 b d_{2} m^{2}\left(2 p r+q^{2}\right)}{\delta}$,

$$
d_{0}=d_{1}=0, k=b m^{3}\left(q^{2}-4 p r\right) .
$$

Case 4: $c_{0}=-\frac{2 b d_{0} m^{2}\left(2 p r+q^{2}\right)}{\delta}, c_{1}=-\frac{12 b d_{0} m^{2} q r}{\delta}, c_{2}=-\frac{12 b d_{0} m^{2} r^{2}}{\delta}$, 


$$
d_{1}=d_{2}=0, k=b m^{3}\left(q^{2}-4 p r\right) .
$$

Avoiding the similar task, we think better to present only the solutions for case 1 which makes available the following exact traveling wave solutions:

For the consideration $q^{2}-4 p r<0$ and $r \neq 0$,

$$
\begin{aligned}
& u_{1}(\xi)=-\frac{3 b m^{2}}{\delta}\left\{2\left(2 p r-q^{2}+q \sqrt{4 p r-q^{2}} \tan \left(\sqrt{4 p r-q^{2}} \xi / 2\right)\right)\right. \\
& \left.-\left(q-\sqrt{4 p r-q^{2}} \tan \left(\sqrt{4 p r-q^{2}} \xi / 2\right)\right)^{2}\right\}, \\
& u_{2}(\xi)=-\frac{3 b m^{2}}{\delta}\left\{2\left(2 p r-q^{2}-q \sqrt{4 p r-q^{2}} \cot \left(\sqrt{4 p r-q^{2}} \xi / 2\right)\right)\right. \\
& \left.-\left(q+\sqrt{4 p r-q^{2}} \cot \left(\sqrt{4 p r-q^{2}} \xi / 2\right)\right)^{2}\right\},
\end{aligned}
$$

where $\xi=\frac{b m^{3}\left(4 p r-q^{2}\right) t^{\alpha}}{\alpha}+\frac{m x^{\beta}}{\beta}$.

When $q^{2}-4 p r>0$ and $r \neq 0$,

$$
\begin{gathered}
u_{3}(\xi)=-\frac{3 b m^{2}}{\delta}\left\{2\left(2 p r-q^{2}-q \sqrt{q^{2}-4 p r} \tanh \left(\sqrt{q^{2}-4 p r} \xi / 2\right)\right)\right. \\
\left.-\left(q+\sqrt{q^{2}-4 p r} \tanh \left(\sqrt{q^{2}-4 p r} \xi / 2\right)\right)^{2}\right\} \\
u_{4}(\xi)=-\frac{3 b m^{2}}{\delta}\left\{2\left(2 p r-q^{2}-q \sqrt{q^{2}-4 p r} \operatorname{coth}\left(\sqrt{q^{2}-4 p r} \xi / 2\right)\right)\right. \\
\left.-\left(q+\sqrt{q^{2}-4 p r} \operatorname{coth}\left(\sqrt{q^{2}-4 p r} \xi / 2\right)\right)^{2}\right\}
\end{gathered}
$$

where $\xi=\frac{b m^{3}\left(4 p r-q^{2}\right) t^{\alpha}}{\alpha}+\frac{m x^{\beta}}{\beta}$.

When $q^{2}+4 p^{2}<0, r \neq 0$ and $r=-p$,

$$
\begin{gathered}
u_{5}(\xi)=\frac{3 b m^{2}}{\delta}\left\{2\left(2 p^{2}+q^{2}-q \sqrt{-q^{2}-4 p^{2}} \tan \left(\sqrt{-q^{2}-4 p^{2}} \xi / 2\right)\right)\right. \\
\left.-\left(q-\sqrt{-q^{2}-4 p^{2}} \tan \left(\sqrt{-q^{2}-4 p^{2}} \xi / 2\right)\right)^{2}\right\}
\end{gathered}
$$




$$
\begin{gathered}
u_{6}(\xi)=\frac{3 b m^{2}}{\delta}\left\{2\left(2 p^{2}+q^{2}+q \sqrt{-q^{2}-4 p^{2}} \cot \left(\sqrt{-q^{2}-4 p^{2}} \xi / 2\right)\right)\right. \\
\left.-\left(q+\sqrt{-q^{2}-4 p^{2}} \cot \left(\sqrt{-q^{2}-4 p^{2}} \xi / 2\right)\right)^{2}\right\},
\end{gathered}
$$

where $\xi=-\frac{b m^{3}\left(4 p^{2}+q^{2}\right) t^{\alpha}}{\alpha}+\frac{m x^{\beta}}{\beta}$.

When $q^{2}+4 p^{2}>0, r \neq 0$ and $r=-p$,

$$
\begin{gathered}
u_{7}(\xi)=\frac{3 b m^{2}}{\delta}\left\{2\left(2 p^{2}+q^{2}+q \sqrt{q^{2}+4 p^{2}} \tanh \left(\sqrt{q^{2}+4 p^{2}} \xi / 2\right)\right)\right. \\
\left.-\left(q+\sqrt{q^{2}+4 p^{2}} \tanh \left(\sqrt{q^{2}+4 p^{2}} \xi / 2\right)\right)^{2}\right\}, \\
u_{8}(\xi)=\frac{3 b m^{2}}{\delta}\left\{2\left(2 p^{2}+q^{2}+q \sqrt{q^{2}+4 p^{2}} \operatorname{coth}\left(\sqrt{q^{2}+4 p^{2}} \xi / 2\right)\right)\right. \\
\left.-\left(q+\sqrt{q^{2}+4 p^{2}} \operatorname{coth}\left(\sqrt{q^{2}+4 p^{2}} \xi / 2\right)\right)^{2}\right\},
\end{gathered}
$$

where $\xi=-\frac{b m^{3}\left(4 p^{2}+q^{2}\right) t^{\alpha}}{\alpha}+\frac{m x^{\beta}}{\beta}$.

When $q^{2}-4 p^{2}<0$ and $r=p$,

$$
\begin{aligned}
u_{9}(\xi)= & -\frac{3 b m^{2}}{\delta}\left\{2\left(2 p^{2}-q^{2}+q \sqrt{-q^{2}+4 p^{2}} \tan \left(\sqrt{-q^{2}+4 p^{2}} \xi / 2\right)\right)\right. \\
& \left.+\left(-q+\sqrt{-q^{2}+4 p^{2}} \tan \left(\sqrt{-q^{2}+4 p^{2}} \xi / 2\right)\right)^{2}\right\}, \\
u_{10}(\xi) & =-\frac{3 b m^{2}}{\delta}\left\{2\left(2 p^{2}-q^{2}-q \sqrt{-q^{2}+4 p^{2}} \tan \left(\sqrt{-q^{2}+4 p^{2}} \xi / 2\right)\right)\right. \\
& \left.+\left(q+\sqrt{-q^{2}+4 p^{2}} \tan \left(\sqrt{-q^{2}+4 p^{2}} \xi / 2\right)\right)^{2}\right\},
\end{aligned}
$$

where $\xi=\frac{b m^{3}\left(4 p^{2}-q^{2}\right) t^{\alpha}}{\alpha}+\frac{m x^{\beta}}{\beta}$.

When $q^{2}-4 p^{2}>0$ and $r=p$,

$$
u_{11}(\xi)=-\frac{3 b m^{2}}{\delta}\left\{2\left(2 p^{2}-q^{2}-q \sqrt{q^{2}-4 p^{2}} \tanh \left(\sqrt{q^{2}-4 p^{2}} \xi / 2\right)\right)\right.
$$




$$
\begin{gathered}
\left.+\left(-q-\sqrt{q^{2}-4 p^{2}} \tan h\left(\sqrt{q^{2}-4 p^{2}} \xi / 2\right)\right)^{2}\right\} \\
u_{12}(\xi)=-\frac{3 b m^{2}}{\delta}\left\{2\left(2 p^{2}-q^{2}-q \sqrt{q^{2}-4 p^{2}} \operatorname{coth}\left(\sqrt{q^{2}-4 p^{2}} \xi / 2\right)\right)\right. \\
\left.+\left(-q-\sqrt{q^{2}-4 p^{2}} \operatorname{coth}\left(\sqrt{q^{2}-4 p^{2}} \xi / 2\right)\right)^{2}\right\},
\end{gathered}
$$

where $\xi=\frac{b m^{3}\left(4 p^{2}-q^{2}\right) t^{\alpha}}{\alpha}+\frac{m x^{\beta}}{\beta}$.

When $r p<0, q=0$ and $r \neq 0$,

$$
\begin{aligned}
& u_{13}(\xi)=-12 b m^{2} r p \operatorname{sech}^{2}(\sqrt{-r p} \xi) / \delta, \\
& u_{14}(\xi)=12 b m^{2} \operatorname{prcosech}^{2}(\sqrt{-r p} \xi) / \delta,
\end{aligned}
$$

where $\xi=\frac{4 p r b m^{3} t^{\alpha}}{\alpha}+\frac{m x^{\beta}}{\beta}$.

When $q=0$ and $p=-r$,

$$
u_{15}(\xi)=-\frac{48 b m^{2} r^{2} e^{2 r \xi}}{\delta\left(1-e^{2 r \xi}\right)^{2}}
$$

where $\xi=-\frac{4 b m^{3} r^{2} t^{\alpha}}{\alpha}+\frac{m x^{\beta}}{\beta}$.

When $q=r=K$ and $p=0$,

$$
u_{16}(\xi)=-\frac{12 b m^{2} K^{2} e^{K \xi}}{\delta\left(1-e^{K \xi}\right)^{2}}
$$

where $\xi=-\frac{b m^{3} K^{2} t^{\alpha}}{\alpha}+\frac{m x^{\beta}}{\beta}$.

When $q=p+r$,

$$
u_{17}(\xi)=-\frac{12 b m^{2} r}{\delta} \times \frac{(p-r)^{2} e^{(p-r) \xi}}{\left(1-r e^{(p-r) \xi}\right)^{2}},
$$

where $\xi=-\frac{b m^{3}(p-r)^{2} t^{\alpha}}{\alpha}+\frac{m x^{\beta}}{\beta}$. 
When $q=-(p+r)$,

$$
u_{18}(\xi)=-\frac{12 b m^{2} r}{\delta} \times \frac{(p-r)^{2} e^{(p-r) \xi}}{\left(p-r e^{(p-r) \xi}\right)^{2}},
$$

where $\xi=-\frac{b m^{3}(p-r)^{2} t^{\alpha}}{\alpha}+\frac{m x^{\beta}}{\beta}$.

When $p=0$,

$$
u_{19}(\xi)=-\frac{12 b m^{2} r q^{2} e^{q \xi}}{\delta\left(1-r e^{q \xi}\right)^{2}}
$$

where $\xi=-\frac{b m^{3} q^{2} t^{\alpha}}{\alpha}+\frac{m x^{\beta}}{\beta}$.

When $r=q=p \neq 0$,

$$
u_{20}(\xi)=-9 b m^{2} p^{2} \sec ^{2}(\sqrt{3} p \xi / 2) / \delta,
$$

where $\xi=\frac{3 b m^{3} p^{2} t^{\alpha}}{\alpha}+\frac{m x^{\beta}}{\beta}$.

When $r=p$ and $q=0$,

$$
u_{21}(\xi)=-12 b m^{2} p^{2} \sec ^{2}(p \xi) / \delta,
$$

where $\xi=\frac{4 b m^{3} p^{2} t^{\alpha}}{\alpha}+\frac{m x^{\beta}}{\beta}$.

We hunt the earlier recorded results for the nonlinear $\mathrm{KdV}$ equation and conclude that our gained outcomes are different and novel [39-43].

\section{The time and space fractional nonlinear WBBM equation}

The integer order WBBM equation has been derived by Wazwaz in 2017 which is a modified form of $\mathrm{BBM}$ equation and introduced as the wide-ranging model for scientific phenomena [47]. Thereafter, Seadawy et al. (2019) has considered the equation to be fractional order [45]. This equation is given as follows [44]: 


$$
D_{t}^{\alpha} u+D_{x}^{\alpha} u+D_{y}^{\alpha} u^{3}-D_{x z t}^{3 \alpha} u=0
$$

Apply the transformation

$$
u(x, y, z, t)=u(\xi), \xi=\frac{1}{\alpha}\left(\iota x^{\alpha}+\rho y^{\alpha}+\sigma z^{\alpha}-\tau t^{\alpha}\right)
$$

to convert Eq. (5.1) into the following ODE

$$
(\iota-\tau) u+\rho\left(u^{3}\right)^{\prime}+\sigma \iota \tau u^{\prime \prime \prime}=0
$$

Integrate this and consider the integral constant to be zero. Accordingly,

$$
(\iota-\tau) u+\rho u^{3}+\sigma \iota \tau u^{\prime \prime}=0
$$

Due to homogenous balance principle, the solution of Eq. (5.4) stands as

$$
u(\xi)=\frac{c_{0}+c_{1} a^{\phi(\xi)}}{d_{0}+d_{1} a^{\phi(\xi)}}
$$

Eq. (5.5) and its derivative with Eq. (2.5) turns the left side of Eq. (5.4) into a polynomial whose coefficients are set to zero and solve for the following results:

Case 1: $c_{0}= \pm \frac{\left(2 p d_{1}-q d_{0}\right) \sqrt{\iota-\tau}}{\sqrt{\rho\left(4 p r-q^{2}\right)}}, c_{1}= \pm \frac{\left(q d_{1}-2 r d_{0}\right) \sqrt{\iota-\tau}}{\sqrt{\rho\left(4 p r-q^{2}\right)}}, \sigma=\frac{2(\tau-\iota)}{\iota \tau\left(4 p r-q^{2}\right)}$

Case 2: $c_{0}= \pm \frac{d_{0} \sqrt{\rho(\iota-\tau)\left(4 p r-q^{2}\right)}}{\rho q}, c_{1}=0, d_{1}=\frac{2 r d_{0}}{q}, \sigma=\frac{2(\tau-\iota)}{\iota \tau\left(4 p r-q^{2}\right)}$

Case 3: $c_{0}= \pm \frac{d_{1} \sqrt{3 \rho(\tau-\iota)}\left(q \pm \sqrt{3 q^{2}-12 p r}\right)}{6 \rho r}, c_{1}= \pm \frac{d_{1} \sqrt{3 \rho(\tau-\iota)}}{3 \rho}$

$$
d_{0}=\frac{d_{1}\left(3 q \pm \sqrt{3 q^{2}-12 p r}\right)}{6 r}, \sigma=\frac{2(\tau-\iota)}{\iota \tau\left(4 p r-q^{2}\right)}
$$

Case 4: $c_{0}= \pm \frac{q d_{0} \sqrt{(\iota-\tau)}}{\sqrt{\rho\left(4 p r-q^{2}\right)}}, c_{1}= \pm \frac{2 r d_{0} \sqrt{(\iota-\tau)}}{\sqrt{\rho\left(4 p r-q^{2}\right)}}, d_{1}=0, \sigma=\frac{2(\tau-\iota)}{\iota \tau\left(4 p r-q^{2}\right)}$

The above cases harvest a lot of solutions which all are not recorded here for simplicity. Accordingly, the traveling wave solutions for case 1 are initiated as follows:

Considering $q^{2}-4 p r<0$ and $r \neq 0$, 


$$
\begin{aligned}
& u_{1,2}(\xi)= \pm \frac{\sqrt{\iota-\tau}}{\left.\sqrt{\rho\left(4 p r-q^{2}\right.}\right)} \times \frac{2 r\left(2 d_{1} p-q d_{0}\right)+\left(q d_{1}-2 r d_{0}\right)\left(-q+\sqrt{4 p r-q^{2}} \tan \left(\sqrt{4 p r-q^{2}} \xi / 2\right)\right)}{2 r d_{0}+d_{1}\left(-q+\sqrt{4 p r-q^{2}} \tan \left(\sqrt{4 p r-q^{2}} \xi / 2\right)\right)}, \\
& u_{3,4}(\xi)= \pm \frac{\sqrt{l-\tau}}{\left.\sqrt{\rho\left(4 p r-q^{2}\right.}\right)} \times \frac{2 r\left(2 d_{1} p-q d_{0}\right)+\left(q d_{1}-2 r d_{0}\right)\left(-q-\sqrt{4 p r-q^{2}} \cot \left(\sqrt{4 p r-q^{2}} \xi / 2\right)\right)}{2 r d_{0}+d_{1}\left(-q-\sqrt{4 p r-q^{2}} \cot \left(\sqrt{4 p r-q^{2}} \xi / 2\right)\right)},
\end{aligned}
$$

where $\xi=\frac{1}{\alpha}\left(\iota x^{\alpha}+\rho y^{\alpha}-\tau t^{\alpha}\right)+\frac{2(\tau-\iota) z^{\alpha}}{\alpha \iota \tau\left(4 p r-q^{2}\right)}$.

According to the statement $q^{2}-4 p r>0$ and $r \neq 0$,

$$
\begin{aligned}
& u_{5,6}(\xi)= \pm \frac{\sqrt{l-\tau}}{\sqrt{\rho\left(4 p r-q^{2}\right)}} \times \frac{2 r\left(2 d_{1} p-q d_{0}\right)+\left(q d_{1}-2 r d_{0}\right)\left(-q-\sqrt{q^{2}-4 p r} \tanh \left(\sqrt{q^{2}-4 p r} \xi / 2\right)\right)}{2 r d_{0}+d_{1}\left(-q-\sqrt{q^{2}-4 p r} \tanh \left(\sqrt{q^{2}-4 p r} \xi / 2\right)\right)}, \\
& u_{7,8}(\xi)= \pm \frac{\sqrt{l-\tau}}{\sqrt{\rho\left(4 p r-q^{2}\right)}} \times \frac{2 r\left(2 d_{1} p-q d_{0}\right)+\left(q d_{1}-2 r d_{0}\right)\left(-q-\sqrt{q^{2}-4 p r} \operatorname{coth}\left(\sqrt{q^{2}-4 p r} \xi / 2\right)\right)}{2 r d_{0}+d_{1}\left(-q-\sqrt{q^{2}-4 p r} \operatorname{coth}\left(\sqrt{q^{2}-4 p r} \xi / 2\right)\right)},
\end{aligned}
$$

where $\xi=\frac{1}{\alpha}\left(\iota x^{\alpha}+\rho y^{\alpha}-\tau t^{\alpha}\right)+\frac{2(\tau-\iota) z^{\alpha}}{\alpha \iota \tau\left(4 p r-q^{2}\right)}$.

For the assumption $q^{2}+4 p^{2}<0, r \neq 0$ and $r=-p$,

$$
\begin{aligned}
& u_{9,10}(\xi)= \pm \frac{\sqrt{l-\tau}}{\left.\sqrt{\rho\left(-4 p^{2}-q^{2}\right.}\right)} \times \frac{-2 p\left(2 d_{1} p-q d_{0}\right)+\left(q d_{1}+2 p d_{0}\right)\left(q-\sqrt{-q^{2}-4 p^{2}} \tan \left(\sqrt{-q^{2}-4 p^{2}} \xi / 2\right)\right)}{-2 p d_{0}+d_{1}\left(q-\sqrt{-q^{2}-4 p^{2}} \tan \left(\sqrt{-q^{2}-4 p^{2}} \xi / 2\right)\right)} \\
& u_{11,12}(\xi)= \pm \frac{\sqrt{l-\tau}}{\sqrt{\rho\left(-4 p^{2}-q^{2}\right)}} \times \frac{-2 p\left(2 d_{1} p-q d_{0}\right)+\left(q d_{1}+2 p d_{0}\right)\left(q+\sqrt{-q^{2}-4 p^{2}} \cot \left(\sqrt{-q^{2}-4 p^{2}} \xi / 2\right)\right)}{-2 p d_{0}+d_{1}\left(q+\sqrt{-q^{2}-4 p^{2}} \cot \left(\sqrt{-q^{2}-4 p^{2}} \xi / 2\right)\right)}
\end{aligned}
$$

where $\xi=\frac{1}{\alpha}\left(\iota x^{\alpha}+\rho y^{\alpha}-\tau t^{\alpha}\right)-\frac{2(\tau-\iota) z^{\alpha}}{\alpha \iota \tau\left(4 p^{2}+q^{2}\right)}$.

If we consider $q^{2}+4 p^{2}>0, r \neq 0$ and $r=-p$,

$$
\begin{aligned}
& u_{13,14}(\xi)= \pm \frac{\sqrt{l-\tau}}{\sqrt{\rho\left(-4 p^{2}-q^{2}\right)}} \times \frac{-2 p\left(2 d_{1} p-q d_{0}\right)+\left(q d_{1}+2 p d_{0}\right)\left(q+\sqrt{q^{2}+4 p^{2}} \tanh \left(\sqrt{q^{2}+4 p^{2}} \xi / 2\right)\right)}{-2 p d_{0}+d_{1}\left(q+\sqrt{q^{2}+4 p^{2}} \tanh \left(\sqrt{q^{2}+4 p^{2}} \xi / 2\right)\right)} \\
& u_{15,16}(\xi)= \pm \frac{\sqrt{l-\tau}}{\sqrt{\rho\left(-4 p^{2}-q^{2}\right)}} \times \frac{-2 p\left(2 d_{1} p-q d_{0}\right)+\left(q d_{1}+2 p d_{0}\right)\left(q+\sqrt{q^{2}+4 p^{2}} \operatorname{coth}\left(\sqrt{q^{2}+4 p^{2}} \xi / 2\right)\right)}{-2 p d_{0}+d_{1}\left(q+\sqrt{q^{2}+4 p^{2}} \operatorname{coth}\left(\sqrt{q^{2}+4 p^{2}} \xi / 2\right)\right)}
\end{aligned}
$$

where $\xi=\frac{1}{\alpha}\left(\iota x^{\alpha}+\rho y^{\alpha}-\tau t^{\alpha}\right)-\frac{2(\tau-\iota) z^{\alpha}}{\alpha \iota \tau\left(4 p^{2}+q^{2}\right)}$. 
On one occasion $q^{2}-4 p^{2}<0$ and $r=p$,

$$
\begin{aligned}
& u_{17,18}(\xi)= \pm \frac{\sqrt{l-\tau}}{\left.\sqrt{\rho\left(4 p^{2}-q^{2}\right.}\right)} \times \frac{2 p\left(2 d_{1} p-q d_{0}\right)+\left(q d_{1}-2 p d_{0}\right)\left(-q+\sqrt{4 p^{2}-q^{2}} \tan \left(\sqrt{4 p^{2}-q^{2}} \xi / 2\right)\right)}{2 p d_{0}+d_{1}\left(-q+\sqrt{4 p^{2}-q^{2}} \tan \left(\sqrt{4 p^{2}-q^{2}} \xi / 2\right)\right)} \\
& u_{19,20}(\xi)= \pm \frac{\sqrt{l-\tau}}{\sqrt{\rho\left(4 p^{2}-q^{2}\right)}} \times \frac{2 p\left(2 d_{1} p-q d_{0}\right)+\left(q d_{1}-2 p d_{0}\right)\left(-q-\sqrt{4 p^{2}-q^{2}} \cot \left(\sqrt{4 p^{2}-q^{2}} \xi / 2\right)\right)}{2 p d_{0}+d_{1}\left(-q-\sqrt{4 p^{2}-q^{2}} \cot \left(\sqrt{4 p^{2}-q^{2}} \xi / 2\right)\right)}
\end{aligned}
$$

where $\xi=\frac{1}{\alpha}\left(\iota x^{\alpha}+\rho y^{\alpha}-\tau t^{\alpha}\right)+\frac{2(\tau-\iota) z^{\alpha}}{\alpha \iota \tau\left(4 p^{2}-q^{2}\right)}$.

Following the condition $q^{2}-4 p^{2}>0$ and $r=p$,

$$
\begin{aligned}
& u_{21,22}(\xi)= \pm \frac{\sqrt{\iota-\tau}}{\sqrt{\rho\left(4 p^{2}-q^{2}\right)}} \times \frac{2 p\left(2 d_{1} p-q d_{0}\right)+\left(q d_{1}-2 p d_{0}\right)\left(-q-\sqrt{q^{2}-4 p^{2}} \tanh \left(\sqrt{q^{2}-4 p^{2}} \xi / 2\right)\right)}{2 p d_{0}+d_{1}\left(-q-\sqrt{q^{2}-4 p^{2}} \tanh \left(\sqrt{q^{2}-4 p^{2}} \xi / 2\right)\right)} \\
& u_{23,24}(\xi)= \pm \frac{\sqrt{l-\tau}}{\sqrt{\rho\left(4 p^{2}-q^{2}\right)}} \times \frac{2 p\left(2 d_{1} p-q d_{0}\right)+\left(q d_{1}-2 p d_{0}\right)\left(-q-\sqrt{q^{2}-4 p^{2}} \operatorname{coth}\left(\sqrt{q^{2}-4 p^{2}} \xi / 2\right)\right)}{2 p d_{0}+d_{1}\left(-q-\sqrt{q^{2}-4 p^{2}} \operatorname{coth}\left(\sqrt{q^{2}-4 p^{2}} \xi / 2\right)\right)}
\end{aligned}
$$

where $\xi=\frac{1}{\alpha}\left(\iota x^{\alpha}+\rho y^{\alpha}-\tau t^{\alpha}\right)+\frac{2(\tau-\iota) z^{\alpha}}{\alpha \iota \tau\left(4 p^{2}-q^{2}\right)}$.

Under the assessment $r p<0, q=0$ and $r \neq 0$,

$$
\begin{aligned}
& u_{25,26}(\xi)= \pm \frac{\sqrt{l-\tau}}{\sqrt{\rho p r}} \times \frac{d_{1} p+r d_{0} \sqrt{-p / r} \tanh (\sqrt{-r p} \xi)}{d_{0}-d_{1} \sqrt{-p / r} \tanh (\sqrt{-r p} \xi)}, \\
& u_{27,28}(\xi)= \pm \frac{\sqrt{l-\tau}}{\sqrt{\rho p r}} \times \frac{d_{1} p+r d_{0} \sqrt{-p / r} \operatorname{coth}(\sqrt{-r p} \xi)}{d_{0}-d_{1} \sqrt{-p / r} \operatorname{coth}(\sqrt{-r p} \xi)}
\end{aligned}
$$

where $\xi=\frac{1}{\alpha}\left(\iota x^{\alpha}+\rho y^{\alpha}-\tau t^{\alpha}\right)+\frac{(\tau-\iota) z^{\alpha}}{2 \alpha \iota \tau p r}$.

For the assumption $q=0$ and $p=-r$,

$$
u_{29,30}(\xi)= \pm \frac{\sqrt{l-\tau}}{\sqrt{-\rho}} \times \frac{d_{0}+d_{1}+\left(d_{0}-d_{1}\right) e^{2 r \xi}}{d_{0}+d_{1}+\left(d_{1}-d_{0}\right) e^{2 r \xi}}
$$

where $\xi=\frac{1}{\alpha}\left(\iota x^{\alpha}+\rho y^{\alpha}-\tau t^{\alpha}\right)-\frac{(\tau-\iota) z^{\alpha}}{2 \alpha \iota \tau r^{2}}$.

With the condition $p=q=K$ and $r=0$, 


$$
u_{31,32}(\xi)= \pm \frac{\sqrt{l-\tau}}{\sqrt{-\rho}} \times \frac{-d_{0}+d_{1}\left(1+e^{K \xi}\right)}{d_{0}+d_{1}\left(e^{K \xi}-1\right)}
$$

where $\xi=\frac{1}{\alpha}\left(\iota x^{\alpha}+\rho y^{\alpha}-\tau t^{\alpha}\right)-\frac{2(\tau-\iota) z^{\alpha}}{\alpha \iota \tau K^{2}}$.

When $q=r=K$ and $p=0$,

$$
u_{33,34}(\xi)= \pm \frac{\sqrt{l-\tau}}{\sqrt{-\rho}} \times \frac{d_{1}\left(1-e^{K \xi}\right)-d_{0}\left(1+e^{K \xi}\right)}{d_{0}\left(1-e^{K \xi}\right)+d_{1} e^{K \xi}}
$$

where $\xi=\frac{1}{\alpha}\left(\iota x^{\alpha}+\rho y^{\alpha}-\tau t^{\alpha}\right)-\frac{2(\tau-\iota) z^{\alpha}}{\alpha \iota \tau K^{2}}$.

If we assign $q=p+r$,

$$
u_{35,36}(\xi)= \pm \frac{\sqrt{\iota-\tau}}{(p-r) \sqrt{-\rho}} \times \frac{\left(2 d_{1} p-(p+r) d_{0}\right)\left(1-r e^{(p-r) \xi}\right)-\left((p+r) d_{1}-2 r d_{0}\right)\left(1-p e^{(p-r) \xi}\right)}{d_{0}\left(1-r e^{(p-r) \xi}\right)-d_{1}\left(1-p e^{(p-r) \xi}\right)}
$$

where $\xi=\frac{1}{\alpha}\left(\iota x^{\alpha}+\rho y^{\alpha}-\tau t^{\alpha}\right)-\frac{2(\tau-\iota) z^{\alpha}}{\alpha \iota \tau(p-r)^{2}}$.

Assigning $q=-(p+r)$,

$$
u_{37,38}(\xi)= \pm \frac{\sqrt{l-\tau}}{(p-r) \sqrt{-\rho}} \times \frac{\left(2 d_{1} p+(p+r) d_{0}\right)\left(r-e^{(p-r) \xi}\right)-\left((p+r) d_{1}+2 r d_{0}\right)\left(p-e^{(p-r) \xi}\right)}{\left(d_{0} r+d_{1} p\right)-\left(d_{0}+d_{1}\right) e^{(p-r) \xi}}
$$

where $\xi=\frac{1}{\alpha}\left(\iota x^{\alpha}+\rho y^{\alpha}-\tau t^{\alpha}\right)-\frac{2(\tau-\iota) z^{\alpha}}{\alpha \iota \tau(p-r)^{2}}$.

For $p=0$,

$$
u_{39,40}(\xi)= \pm \frac{\sqrt{\iota-\tau}}{q \sqrt{-\rho}} \times \frac{-q d_{0}\left(1-r e^{q \xi}\right)+\left(q d_{1}-2 r d_{0}\right) q e^{q \xi}}{d_{0}-\left(d_{0} r-d_{1} q\right) e^{q \xi}}
$$

where $\xi=\frac{1}{\alpha}\left(\iota x^{\alpha}+\rho y^{\alpha}-\tau t^{\alpha}\right)-\frac{2(\tau-\iota) z^{\alpha}}{\alpha \iota \tau q^{2}}$.

Fixing $r=q=p \neq 0$,

$$
u_{41,42}(\xi)= \pm \frac{\sqrt{l-\tau}}{p \sqrt{3 \rho}} \times \frac{2\left(2 d_{1} p-p d_{0}\right)+\left(p d_{1}-2 p d_{0}\right)\{\sqrt{3} \tan (\sqrt{3} p \xi / 2)-1\}}{2 d_{0}+d_{1}\{\sqrt{3} \tan (\sqrt{3} p \xi / 2)-1\}}
$$

where $\xi=\frac{1}{\alpha}\left(\iota x^{\alpha}+\rho y^{\alpha}-\tau t^{\alpha}\right)+\frac{2(\tau-\iota) z^{\alpha}}{3 \alpha \iota \tau p^{2}}$. 
According to the setting $r=p$ and $q=0$,

$$
u_{43,44}(\xi)= \pm \frac{\sqrt{l-\tau}}{\sqrt{\rho}} \times \frac{d_{1}-d_{0} \tan (p \xi)}{d_{0}+d_{1} \tan (p \xi)}
$$

where $\xi=\frac{1}{\alpha}\left(\iota x^{\alpha}+\rho y^{\alpha}-\tau t^{\alpha}\right)+\frac{(\tau-\imath) z^{\alpha}}{2 \alpha \iota \tau p^{2}}$.

If we assign $r=0$,

$$
u_{45,46}(\xi)= \pm \frac{\sqrt{l-\tau}}{q \sqrt{-\rho}} \times \frac{\left(2 d_{1} p-q d_{0}\right)+q d_{1}\left(e^{q \xi}-m / n\right)}{d_{0}+d_{1}\left(e^{q \xi}-m / n\right)}
$$

where $\xi=\frac{1}{\alpha}\left(\iota x^{\alpha}+\rho y^{\alpha}-\tau t^{\alpha}\right)-\frac{2(\tau-\iota) z^{\alpha}}{\alpha \iota \tau q^{2}}$.

The above obtained solutions to the WBBM equation are compared with those available in the earlier study and claimed to be recorded in the literature for the first time $[44,45]$.

\section{Graphical appearances of found solutions}

The achieved solutions of the fractional nonlinear Schrodinger equation, KdV equation and WBBM equation are figured out for their physical appearances. Different shapes of solitons like kink shape, anti-kink shape, bell shape, anti-bell shape, peakon, compacton and periodic are brought out graphically. A few graphs are given below:

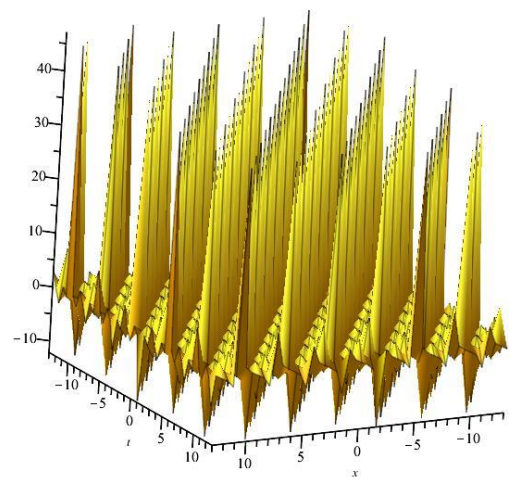

Fig 1: Outline of solution (3.6) for $\alpha=k=p=1$, $q=4, b=m=-2, d_{0}=0, d_{1}=r=5$ in the interval $-13 \leq x, t \leq 13$.

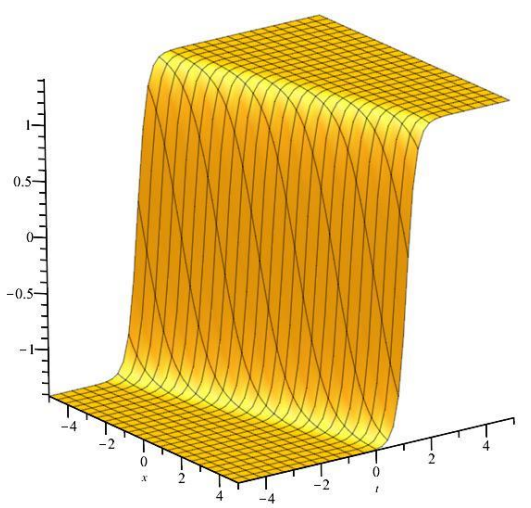

Fig 2: Shape of solution (3.20) for $\alpha=1, r=2$, $d_{0}=-0.2, d_{1}=0.5, k=p=-1, b=-2, m=$ -7 within $-5 \leq x, t \leq 5$. 


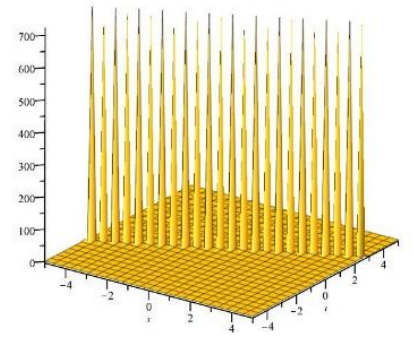

Fig 3: Plot of solution (3.22) for $\alpha=1, d_{1}=K=2$, $d_{0}=-5, k=-1, m=-3, b=-2$ within the interval $-5 \leq x, t \leq 5$.

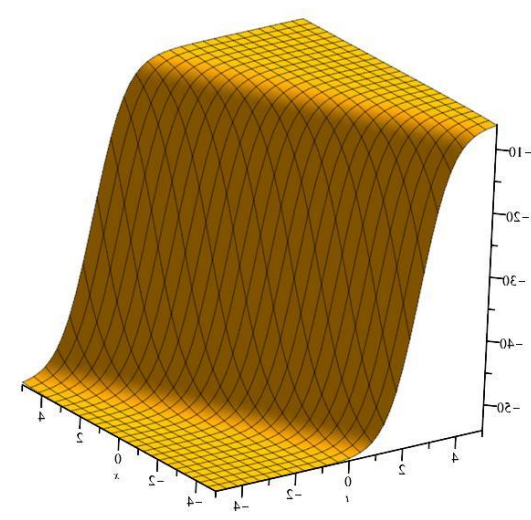

Fig 5: Outline of solution (4.8) for $\alpha=\beta=p=1$, $b=-2, m=0.5, q=4, r=3, \delta=2$ within the range $-5 \leq x, t \leq 5$.

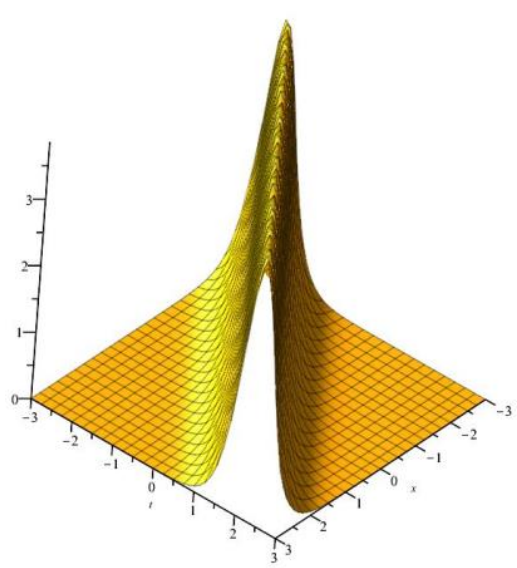

Fig 7: Plot of solution (4.16) for $\alpha=\beta=\delta=m=$ $1, b=1 / 7, p=2, q=5, \delta=1$ in the range $-3 \leq$ $x, t \leq 3$.

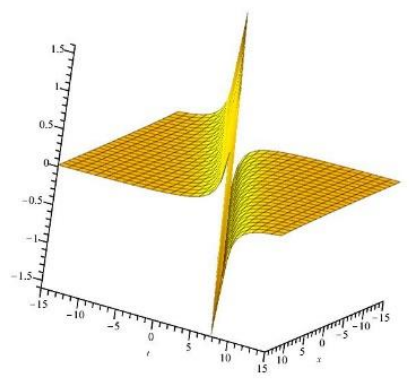

Fig 4: Sketch of solution (3.28) for $\alpha=1, d_{1}=p=$ $2, k=m=-1, b=-2, d_{0}=0$ in the interval $-15 \leq x, t \leq 15$.

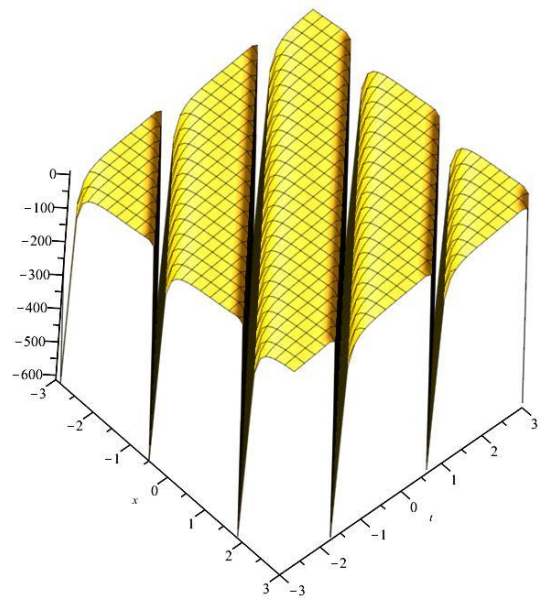

Fig 6: Plot of solution (4.14) for $\alpha=\beta=m=1$, $b=1 / 7, p=2, q=3, \delta=-1$ in the interval $-3 \leq x, t \leq 3$.

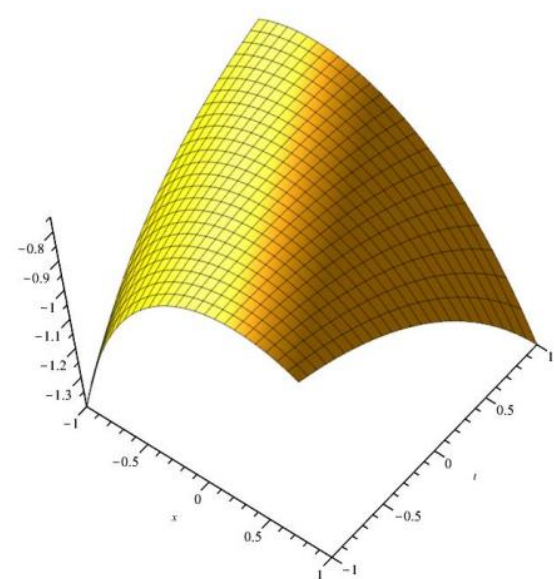

Fig 8: Outline of solution (4.26) for $\alpha=\beta=p=1$, $b=m=0.5, \delta=2$ within the interval $-1 \leq$ $x, t \leq 1$. 

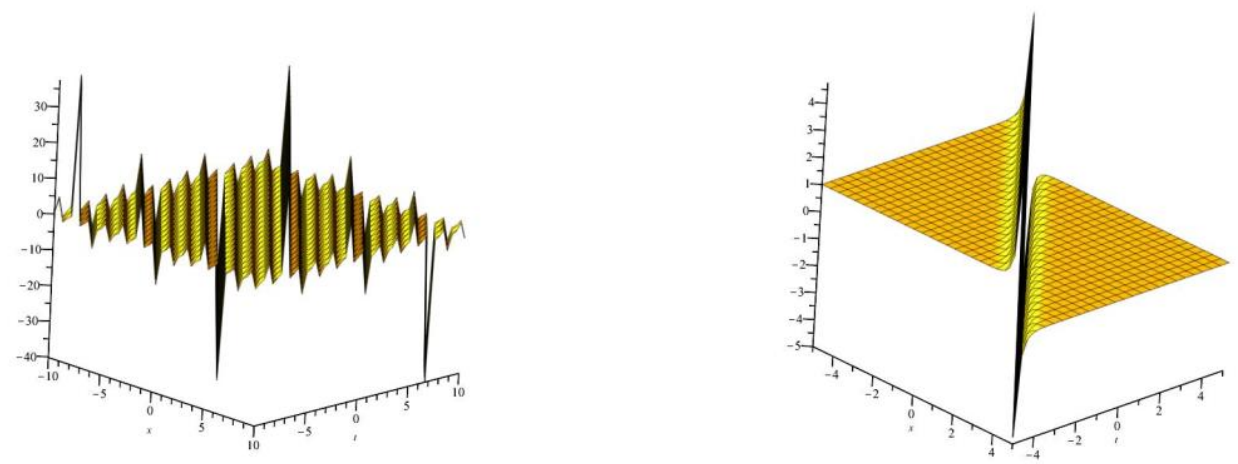

Fig 9: Shape of solution (5.6) for $\alpha=p=1, \tau=$ Fig 10: Outline of solution (5.9) for $\alpha=p=1, \tau=$ $-2, q=\rho=4, r=5, d_{0}=d_{1}=0.5, \iota=2, y=-2, q=4, \rho=-4, r=3, d_{0}=0.5, d_{1}=-0.5$ $z=0$ in $-10 \leq x, t \leq 10$. $\iota=2, y=z=0$ in $-5 \leq x, t \leq 5$.
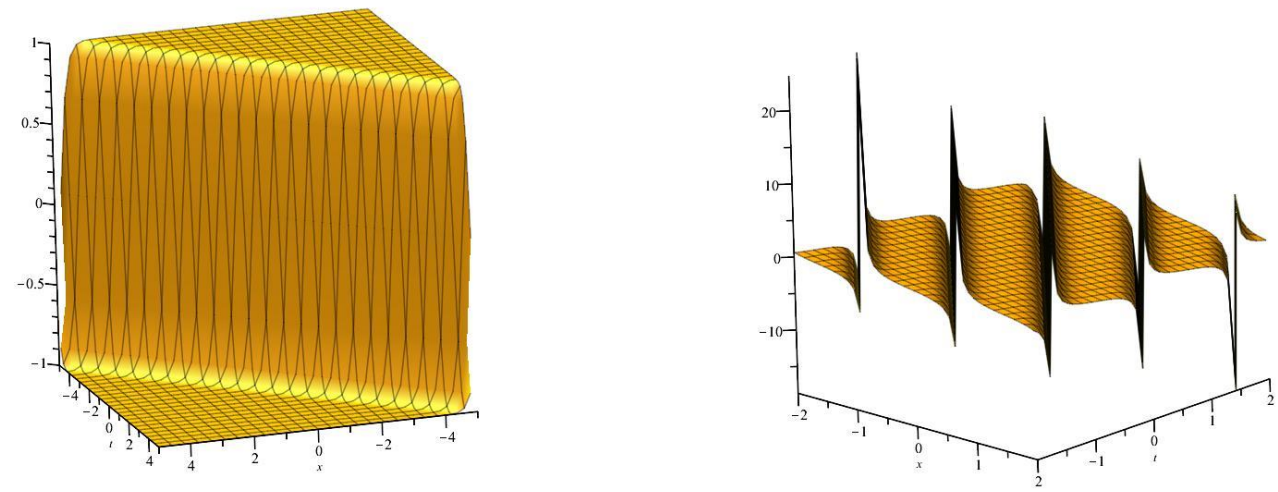

Fig 11: Sketch of solution (5.17) for $\alpha=p=1, \tau=$ $-2, q=4, \rho=-4, d_{0}=d_{1}=-0.2, \iota=2, y=$ $z=0$ in $-5 \leq x, t \leq 5$.
Fig 12: Outline of solution (5.27) for $\alpha=\iota=1, \rho=$ $4, \tau=-1, d_{0}=-2, d_{1}=3, p=2, y=z=0$ in $-2 \leq x, t \leq 2$.

\section{Conclusions}

Our goal was to explore new solitons patterns of the fractional nonlinear Schrodinger equation, Korteweg-De Vries (KdV) equation and the Wazwaz-Benjamin-Bona-Mahony (WBBM) equation. Abundant exact analytic traveling wave solutions to the recommended equations are successfully constructed by executing the proposed improved auxiliary equation technique. This method is efficient, concise and inventive which might be put forwarded for further use to look for soliton and other types solutions of any nonlinear fractional models relating to physical sciences. The obtained solutions cover much more free constants and claim to be new, 
interesting and significant which might be useful to depict the underlying structures of intricate behavior of nature world. So far, we hunt the literature the gained outcomes might newly be visible in the research field and inspire the scientists and scholars to advance the related work in future.

\section{Author Statement}

Md. Tarikul Islam: Conceptualization, Methodology, Writing-Original draft, Supervision; J.F. Gómez-Aguilar: Conceptualization, Methodology, Writing-Original draft preparation, Supervision; Md. Ali Akbar: Conceptualization, Methodology, Writing-Original draft preparation. All authors read and approved the final manuscript.

\section{Acknowledgments}

José Francisco Gómez Aguilar acknowledges the support provided by CONACyT: cátedras CONACyT para jóvenes investigadores 2014 and SNI-CONACyT.

\section{References}

1. K. B. Oldham, J. Spanier, The Fractional Calculus, Academic Press, New York, NY, USA, 1974.

2. C. Rogers, W. F. Shadwick. Backlund transformations and their applications. Vol. 161 of Mathematics in Science and Engineering, Academic Press, New York, USA, 1982.

3. M. J. Ablowitz, P. A. Clarkson. Solitons, Nonlinear Evolution Equations and Inverse Scattering Transform. Cambridge University Press, Cambridge, UK, 1991.

4. K. S. Miller, B. Ross. An Introduction to the Fractional Calculus and Fractional Differential Equations. John Wiley \& Sons, New York, NY, USA, 1993.

5. I. Podlubny. Fractional Differential Equations. vol. 198 of Mathematics in Science and Engineering, Academic Press, San Diego, Calif, USA, 1999. 
6. A. M. Wazwaz. Partial differential equations: Method and applications. Taylor and Francis, 2002.

7. S. W. Yao, S. M. Zekavatmand, H. Rezazadeh, J. Vahidi, M. B. Chaemi and M. Inc. The solitary waves solutions to the Klein-Gordon-Zakharov equations by extended rational methods. AIP Adv., 11 (2021), 065218.

8. N. Maarouf and K. Hilal. Invariant analysis, analytical solutions, and conservation laws for two-dimensional time fractional Fokker-Planck equation. J. Fun. Spaces, 2021 (2021), 249,0392 .

9. M. T. Islam and M. A. Akter. Distinct solutions of nonlinear space-time fractional evolution equations appearing in mathematical physics via a new technique. Partial Diff. Eq. Appl. Math., 3 (2021).

10. A. Neirameh and F. Parvaneh. Analytical solitons for the space-time conformable differential equations using two efficient techniques. Adv. Diff. Eq., 2021, 1-28.

11. M. A. Akbar, N. H. M. Ali and M. T. Islam. Multiple closed form solutions to some fractional order nonlinear evolution equations in physics and plasma physics. AIMS Mathematical, 4 (2019), 397-411.

12. H. Durur, E. Ilhan and H. Bulut. Novel complex wave solutions of the (2+1)-dimensional hyperbolic nonlinear Schrodinger equation. Fractal Fract., 41 (2020).

13. A. Bekir, O. A. Guner. Exact solutions of nonlinear fractional differential equations by ( $\left.G^{\prime} / G\right)$-expansion method. Chin. Phys. B, 22 (2013), 404-409.

14. A. Yokus, H. Durur and S. Duran. Simulation and refraction event of complex hyperbolic type solitary wave in plasma and obtical fiber for the perturbed Chen-Lee-Liu equation. 2021. 
15. M. T. Islam, M. A. Akbar and A.K. Azad. A Rational $\left(G^{\prime} / G\right)$-expansion method and its application to the modified KdV-Burgers equation and the (2+1)-dimensional Boussinesq equation. Non. Studies, 6 (2015), 1-11.

16. H. Durur and A. Yokus. Exact solutions of (2+1)-Ablowitz-Kaup-Newell-Segur equation. Appl. Math. Non. Sci., (2020), 1-6.

17. M. Kaplan, O. Unsal and A. Bekir. Exact solutions of nonlinear Schrodinger equation by using symbolic computation. Math. Meth. Appl. Sci., 39, (2016), 2093-2099.

18. H. Y. Martinez, J. F. G. Aguilar and A. Atangana. First integral method for nonlinear differential equations with conformable derivative. Math. Model. Nat. Phenom., 13 (2018), 14.

19. W. X. Ma and J. H. Lee. A transformed rational function method and exact solutions to the $3+1$ dimensional Jimbo-Miwa equation. Chaos Solitons Fractals, 42 (2009), 1356-1363.

20. A. R. Seadawy. New exact solutions for the Kdv equation with higher order nonlinearity by using the variational method. Comput. Math. Appl., 62 (2011), 3741-3755.

21. D. Lu, A. Seadawy, M. Arshad. Application of extended simple equation method on unstable Schrodinger equations. Optic-Int. J. Light Electron. Opt., 140 (2017), 136-144.

22. M. M. A. Khater, A. R. Seadawy and D. Lu. Elliptic and solitary wave solutions for Bogoyavlenskii equations system, couple Boiti-Leon-Pempinelli equations system and time-fractional Cahn-Allen equation. Res. Phys., 7 (2017), 2325-2333.

23. S. Bibi, S. T. Mohyud-Din, U. Khan and N. Ahmed. Khater method for nonlinear Sharma Tasso-Olever (STO) equation of fractional order. Res. Phys., 7 (2017), 4440-4450.

24. R. A. M. Attia, D. Lu and M. M. A. Khater. Structures of new solitary solutions for the Schwarzian Korteweg De Vries equation and (2+1)-Ablowitz-Kaup-Newell-Segur equation. Phys. J., 1 (2018), 234-254. 
25. M. M. A. Khater, A. R. Seadawy and D. Lu. New optical soliton solutions for nonlinear complex fractional Schrodinger equation via new auxiliary equation method and novel ( $\left.G^{\prime} / G\right)$-expansion method. Pramana-J. Phys., 90 (2018), 1-20.

26. H. Rezazadeh, A. Korkmaz, M. Eslami, S. M. M. Alizamini. A large family of optical solutions to Kundu-Eckhaus model by a new auxiliary equation method. Opt. Quan. Elec., $51(2019), 1-12$.

27. M. M. A. Khater, R. A. M. Attia and D. Lu. Modified auxiliary equation method versus three nonlinear fractional biological models in present explicit wave solutions. Math. Com. Appl., 24 (2019).

28. A. A. Alderemy, R. A. M. Attia, J. F. Alzaidi, D. Lu and M. M. A. Khater. Analytical and semi-analytical wave solutions for longitudinal wave equation via modified auxiliary equation method and Adomian Decomposition method. Thermal Sci., 23 (2019), 19431957.

29. M. M. A. Khater, D. Lu and R. A. M. Attia. Dispersive long wave of nonlinear fractional Wu-Zhang system via a modified auxiliary equation method. AIP Adv., 9 (2019), 025003.

30. R. A. M. Attia, D. Lu and M. M. A. Khater. Chaos and relativistic energy-momentum of the nonlinear time fractional duffing equation. Math. Com. Appl., 24 (2019), 10.

31. M. M. A. Khater, A. A. Mousa, M. A. El-Shorbagy and R. A. M. Attia. Abundant novel wave solutions of nonlinear Klein-Gordon-Zakharov (KGZ) model. Eur. Phys. J. Plus, 136 (2021), 1-11.

32. K. M. Hemida, K. A. Gepreel and M. S. Mohamed. Analytical approximate solution to the time-space nonlinear partial fractional differential equations. Int. J. Pure Appl. Math., 78 (2012), 233-243.

33. M. Kaplan, O. Unsal and A. Bekir. Exact solutions of nonlinear Schrodinger equation by using symbolic computation. Math. Meth. Appl. Sci., 39, (2016). 
34. E. A-B. A. Salam, E. Yousif and M. El-Aasser. Analytical solution of the space-time fractional nonlinear Schrodinger equation. Rep. Math. Phys., 77 (2016), 19-34.

35. Y. Pandir and H. H. Duzgun. New exact solutions of the space-time fractional cubic Schrodinger equation using the new type F-expansion method. Waves Ran. Com. Med., 29 (2019), 425-434.

36. A. M. Wazwaz and L. Kaur. Optical solitons for nonlinear Schrodinger (NLS) equation in normal dispersive regimes. Optik-Int. J. Light Elect. Opt., 184 (2019), 428-435.

37. N. Cheema and M. Younis. New and more general traveling wave solutions for nonlinear Schrodinger equation. Waves Ran. Com. Med., 26 (2016), 30-41.

38. M. A. Akbar, N. H. M. Ali and T. Tanjim. Outset of multiple soliton solutions to the nonlinear Schrodinger equation and the coupled Burgers equations. J. Phys. Commun., 3 (2019), 095013.

39. H. C. Yaslan and A. Girgin. New exact solutions for the conformable space-time fractional KdV, CDG, (2+1)-dimensional CBS and (2+1)-dimensional AKNS equations. J. Taibah Uni. Sci., 13 (2019), 1-8.

40. Z. Odibat. A Riccati equation approach and travelling wave solutions for nonlinear evolution equations. Int. J. Appl. Comput. Math., 3 (2017), 1-13.

41. H. K. Jassim and Dumitru Baleanu. A novel approach for Korteweg-de Vries equation of fractional order. J. Appl. Comput. Mech., 5 (2019), 192-198.

42. J. Liu and Y. Zhang. Analytical study of exact solutions of the nonlinear Korteweg-de Vries equation with space-time fractional derivatives. Mod. Phys. Lett. B, 32 (2018), 1850012.

43. A. Dascioglu, S. Culha and D. V. Bayram. New analytical solutions of the space fractional KdV equation in terms of Jacobi elliptic functions. New Trends Math. Sci., 5 (2017), 232241. 
44. U. Akram, A. R. Seadawy, S. T. R. Rizvi, M. Younis, S. Althobaiti and S. Sayed. Traveling wave solutions for the fractional Wazwaz-Benjamin-Bona-Mahony model in arising shallow water waves. Res. Phys., 20 (2021), 103725.

45. A. R. Seadawy, K. K. Ali and R. I. Nuruddeen. A variety of soliton solutions for the fractional Wazwaz-Benjamin-Bona-Mahony equations. Res. Phys., 12 (2019), 2234-2241.

46. R. Khalil, M. A. Horani, A. Yousef and M. A. M. Sababheh. A new definition of fractional derivative. J. Comput. Appl. Math. 264 (2014), 65-70.

47. A. M. Wazwaz. Exact soliton and kink solutions for new (3+1)-dimensional nonlinear modified equations of wave propagation. Open Eng., 7 (2017), 169-174. 